\title{
A Diffusion-based Guidance System for Autonomous Agents
}

\author{
J. E. R. Staddon ${ }^{\bullet}$ and Ioan M. Chelaru', \\ Department of Psychology: Experimental, Duke University, Durham, NC 27708
}

\begin{abstract}
Search strategy is an important component of any system that uses autonomous agents to detect and neutralize mines. We describe a simple and efficient search strategy derived from research on the adaptive spatial behavior of animals. Electromagnetic sensor data are processed to obtain a discrete spatial target distribution. The target distribution is used as input for a dynamic diffusion process. The diffusion surface is used by the demining agent to optimize its spatial moves through a hill climbing technique. The agent chooses to move to the position with the highest diffusion surface value. If the same diffusion surface is available to all agents, the system can be scaled to guide an indefinite number of independent, non-interfering agents.
\end{abstract}

Keywords: demining, search, discrete diffusion process, spatial navigation

\section{INTRODUCTION}

An attractive approach to the detection and neutralization of mines is by using autonomous agents (termed robots or, in our case, animats). The detection task to be performed by these agents can be broken down into three subproblems: (a) collection of sensor information; (b) processing of sensor data to maximize the probability of target detection (sensor fusion, filtering, regression analysis, etc.); and (c) optimal guidance of the agent or agents. This paper deals with one approach to subproblem (c), the guidance of mine-detecting robots. We describe ongoing work on a very simple, scaleable guidance scheme based on a search model originally developed to explain the spatial behavior of animals foraging for food. The search algorithm uses a dynamic, diffusion-based rule for stimulus generalization, a well-established psychological principle. The diffusion process can reproduce the qualitative properties of spatial navigation in animals: area-restricted search in the open field, finding shortcuts, barrier learning, spatial "insight" in mazes and radial-maze behavior ${ }^{1,2}$. We believe that a modified version of this model can be used to guide demining robots.

The domain of autonomous navigation is well established in the field of artificial intelligence. There are some powerful algorithms addressed to the navigation problem in an environment with obstacles ${ }^{3,4,5}$. These algorithms use quite complex approaches to find the optimal path guiding a robot to its goal. Our algorithm belongs to the class of spatial navigation algorithms developed from the study of animal behavior ${ }^{6}$. All animals, from the very simplest single-celled creatures, to ants, bees and higher primates, must solve fairly complex spatial problems in order to survive. Searching for food, finding a nest and avoiding predators all involve some degree of spatial learning. The paths that animals follow when scouting potential food sites, for example, are usually close to optimal. Nevertheless, as recent research shows, this robust adaptive behavior is accomplished by surprisingly simple means ${ }^{7}$.

Several interesting spatial-navigation models have been developed by animal behavior researchers ${ }^{7}$. Modeling the spatial behavior of ants ${ }^{8,9}$, bees ${ }^{10,11}$, gerbils ${ }^{12}$, or rats ${ }^{13}$, these algorithms provide some "intelligent" features like a built-in compass, barrier detour and spatial insight in specialized mazes. Two complex and powerful navigation systems that use neural networks, based on animal behavior are presented in ${ }^{14,15}$.

By comparison with other implementations, our diffusion-based algorithm is not perfectly optimal: it does not guarantee the shortest search path, for example. But it is simple and can accomplish useful spatial tasks, like multiple-target finding and barrier detour. It is also scaleable, in the sense that it can easily be extended to an indefinite number of independent but non-interfering agents searching the same space.

In the following sections, we describe in detail the sensor data we used for simulations, our pre-processing algorithm, the spatial search procedure, and, finally the results of some computer simulations.

\footnotetext{
- Further author information -

J.E.R.S. (correspondence): Email: staddon@psych.duke.edu; WWW: http://psych.duke.edu/jers/staddon.htm; Telephone: 919-660-5724, Fax: 919-660-5726

I.M.C.: Email: mircea@ psych.duke.edu; WWW: http://psych.duke.edu/postdocs/chelaru.htm;Telephone: 919-660-5674
} 


\section{SENSOR DATA PRE-PROCESSING}

Since our focus is on the spatial-guidance problem, we assume three things: (a) That each agent knows where it is, i.e., it has an accurate $\mathrm{x}$-y spatial coordinate (we do not consider at this point whether this information is conveyed through landmark learning or, as might be more likely for an artificial agent, through a GPS system). (b) That each agent has access to a variable, $D_{i j}$, that represents mine probability at immediately adjacent locations. (c) That each agent knows whether or not a mine is present at its current location. We recognize that assumption (c) is unrealistic, and future versions will incorporate an algorithm that drives a "dig-no-dig" decision-maker. We also assume that each agent can move in eight directions: N, S, E W, NE, NW, $\mathrm{SE}$ and SW.

In our simulations, we used data from the EM61 High Sensitivity Metal Detector ${ }^{16}$, gathered from a known mine array. The raw data were arrays of real numbers. Each array element is the sensor value at a certain node of a regular spatial grid. The resolution for the grid $\mathrm{X}$-axis was 0.5 meter and for the grid $\mathrm{Y}$-axis was 0.2 meter. A preliminary data analysis showed a moderate number of peaks over a noisy background.

To improve the dynamic range and to aid subsequent processing we used the following simple procedure:

1) Compute the minimum and maximum values of the raw data and scale the data to the interval $[0,1]$ with the equation:

$$
s(i, j)=\frac{z(i, j)-\operatorname{Min}}{\operatorname{Max}-\operatorname{Min}}
$$

where $s(i, j)$ is the scaled data at the $(i, j)$ grid position, $z(i, j)$ is the sensor data, Min is the data minimum sensor data value and Max is the maximum sensor data value;

2) We computed the scaled-data histogram in 64 points of the interval [0,1]; with these data, the histogram has a clear peak at the average background value;

3) We set as threshold the histogram peak value in the interval $[0,1]$;

4) We set all values smaller than the threshold equal to the threshold and again scaled the data to the interval $[0,1]$ using Equation (1); in this case Min is the threshold and Max is 1.

These computations slightly improved the dynamic range and scaled the data for further processing. The next step was to decrease the amount of data needed for agent guidance. This is possible because the processed data are sparse: a moderate number of "target" peaks against a large non-informative background. Under these circumstances, all the animat needs is local information. Therefore,

1) the search area is divided into small, equal areas rectangular regions (cells);

2) the agent looks only at data from adjacent cells; and

3 ) the agent moves to the adjacent cell having the largest mine probability $\left(D_{i j}\right)$ value.

Mine probability will be defined later. For the moment, we want to stress the point that local information about the current neighborhood is enough for spatial guidance of the agent. Thus, we need two kinds of information: high spatial resolution (HSR: the sensor data) and low-spatial resolution (LSR) used to extract the location of potential mines (targets). The HSR information is used to create a set of targets that matches as closely as possible the actual mine locations.

We used the following sensor-data pre-processing to create a target map:

1) we divided the search area into small equal rectangles, termed cells (we used $1 \mathrm{~m} \times 1 \mathrm{~m}$ rectangles);

2) for each cell, we selected the largest sensor value; thus we obtained the LSR information;

3) from the LSR we extracted a set of targets, a target map (as we shall see in the next paragraph);

4) the set of targets was used as input to the dynamic diffusion process that guided our animat(s).

It is possible that there is more than one mine in a given cell. In such cases, since only the largest sensor value is taken into account, we could lose information about some mine locations (Steps 3 and 4, above). As we mentioned, we use the LSR information only for movements between the rectangles. For every rectangle, a detailed sensor data analysis must be made to make the digging decision. 
Using LSR data for guidance reduces the computational resources needed for spatial navigation. If, as we anticipate, the dig-no-dig decision will be made by processing only sensor data from the occupied cell, the total resources needed for the demining task are minimized.

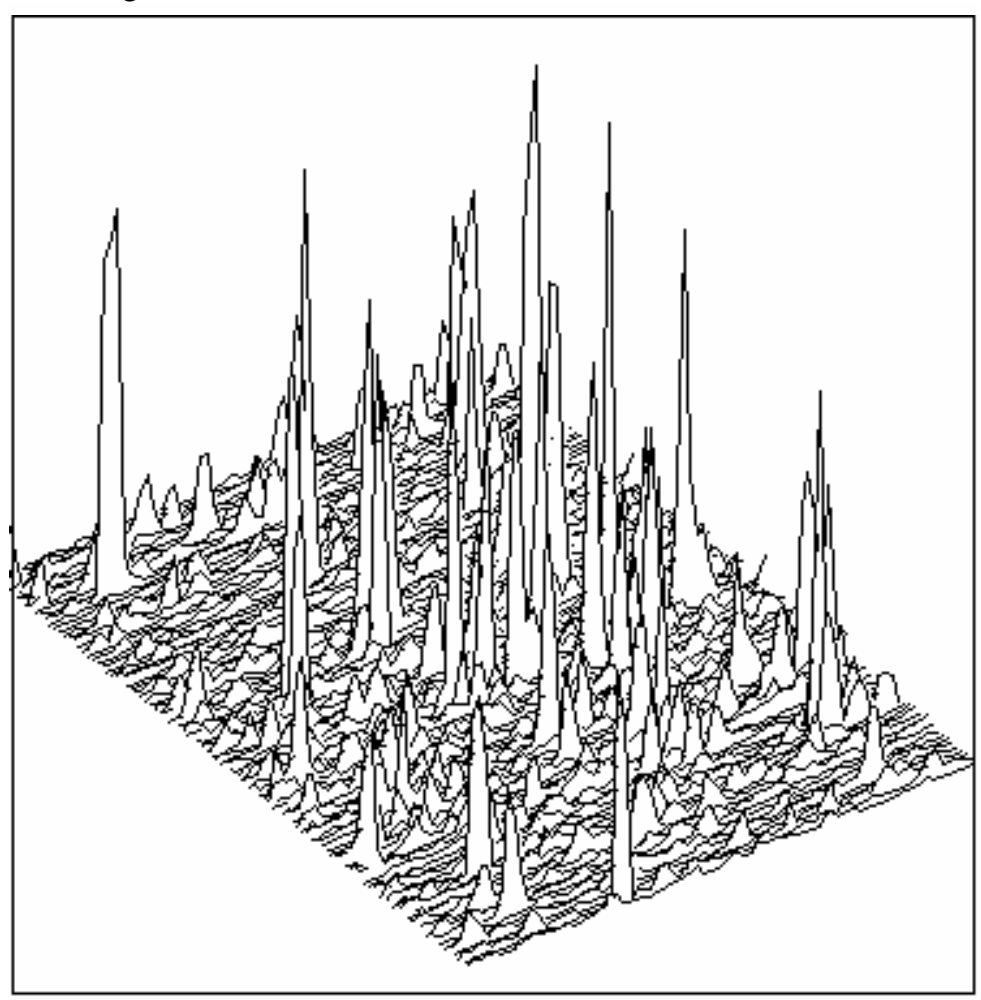

Figure 1. Low-spatial resolution setisor data for site fp20
An example of low-spatial-resolution data (obtained from EM61 High Sensitivity Metal Detector after preprocessing), is given in Figure 1. The site dimensions were $75 \mathrm{~m} \times 100 \mathrm{~m}$, with the sensor data spatial resolutions $0.5 \mathrm{~m}$ on $\mathrm{X}$-axis and $0.2 \mathrm{~m}$ on $\mathrm{Y}$ axis. The number of data samples was more than 75.000 , in the interval $[81,754]$. After pre-processing we had 7676 values, in the interval $[0,1]$, with spatial resolution $1 \mathrm{~m} \times 1 \mathrm{~m}$.

\section{SPATIAL SEARCH}

We assume that the demining agent has a map of the target locations. This map is an array $\mathrm{R}$ of dimension $\mathrm{M} \times \mathrm{N}$, with the elements $r(i, j), \mathrm{i}=1, \ldots, \mathrm{M}, \mathrm{j}=1, \ldots, \mathrm{N}$, where $r(i, j)=1$ if there is a target at position $(\mathrm{i}, \mathrm{j})$, and $r(i, j)=0$ otherwise.

We obtained the array R from the LSR array data by using a threshold between [0,0.5]. All data below the threshold were zeroed and the data above the threshold were made unitary. No doubt, better algorithms could be devised for extracting alarm information from the sensor data, but this was not our primary focus.

The array $\mathrm{R}$ does not represent a true target map because it was obtained from the LSR data. The matrix $\mathrm{R}$ could be interpreted as a map of the regions (rectangles) having the highest probability of mines. In the following, we consider matrix $\mathrm{R}$ as a map of target locations.

From an animal-behavior point of view, the targets given by $\mathrm{R}$ could be considered as food locations, and finding the target positions as a food-searching process in a known environment. Starting with this qualitative similarity, we imagined a search algorithm that uses a discrete two-dimensional diffusion process. Diffusion-based spatial navigation can reproduce qualitative properties of animal spatial behavior like area-restricted search in the open field, finding shortcuts, barrier learning, spatial "insight" in mazes and radial-maze behavior. An autonomous demining agents also needs these capabilities; hence the probable relevance of a diffusion-based system to the demining problem.

The search algorithm is based on a hill-climbing process. The hill is a surface generated by a two-dimensional diffusion process. The diffusion is discrete in time and space. The diffusion grid has the same dimensions as the target position array R.

The value $D_{k}(i, j)$ at the position $(i, j)$ and the iteration $k$, of the diffusion surface is:

$$
D_{k}(i, j)=D_{k-1}(i, j)+\alpha \sum_{V}\left[D_{V, k-1}(i, j)-D_{k-1}(i, j)\right]+r_{k-1}(i, j)
$$

where $V$ is the neighborhood of the position $(i, j), D_{V, k}(i, j)$ is the diffusion value of a neighbor from $V, r_{k}(i, j)$ is the element $(i, j)$ of the target position array at the moment $k$, and $\alpha$ is the diffusion coefficient.

We define the variable $D_{k}(i, j)$ as the mine probability for the cell $(i, j)$ at the moment $k$, and the $D_{V, k}(i, j)$ as the mine probability of a neighbor of the current cell. The term "mine probability" stresses the point that we don't know exactly the meaning (true or false alarm) of the cell target information. The numbers $D_{k}(i, j)$ and $D_{k}(i, j)$ are necessarily estimates, rather than empirical probability values derived from experiment.

Equation (2) has different particular forms depending on the neighborhood type. Thus, for the 8-point neighborhood used in our experiments, Equation (2) has the form: 


$$
\begin{aligned}
D_{k}(i, j)=D_{k-1}(i, j)+ & \alpha\left\{\left[D_{k-1}(i-1, j-1)-D_{k-1}(i, j)\right] / 2+\left[D_{k-1}(i-1, j)-D_{k-1}(i, j)\right]+\right. \\
& +\left[D_{k-1}(i-1, j+1)-D_{k-1}(i, j)\right] / 2+\left[D_{k-1}(i, j-1)-D_{k-1}(i, j)\right]+ \\
& +\left[D_{k-1}(i, j+1)-D_{k-1}(i, j)\right]+\left[D_{k-1}(i+1, j-1)-D_{k-1}(i, j)\right] / 2+ \\
& \left.+\left[D_{k-1}(i+1, j)-D_{k-1}(i, j)\right]+\left[D_{k-1}(i+1, j+1)-D_{k-1}(i, j)\right] / 2\right\}
\end{aligned}
$$

which is the simplest discrete form (Forward Time Continuous Space) of a continuous two-dimensional diffusion process ${ }^{17}$. The diffusion coefficient $\alpha$ must be positive and less than 0.125 in order to have a time-stable discrete diffusion process.

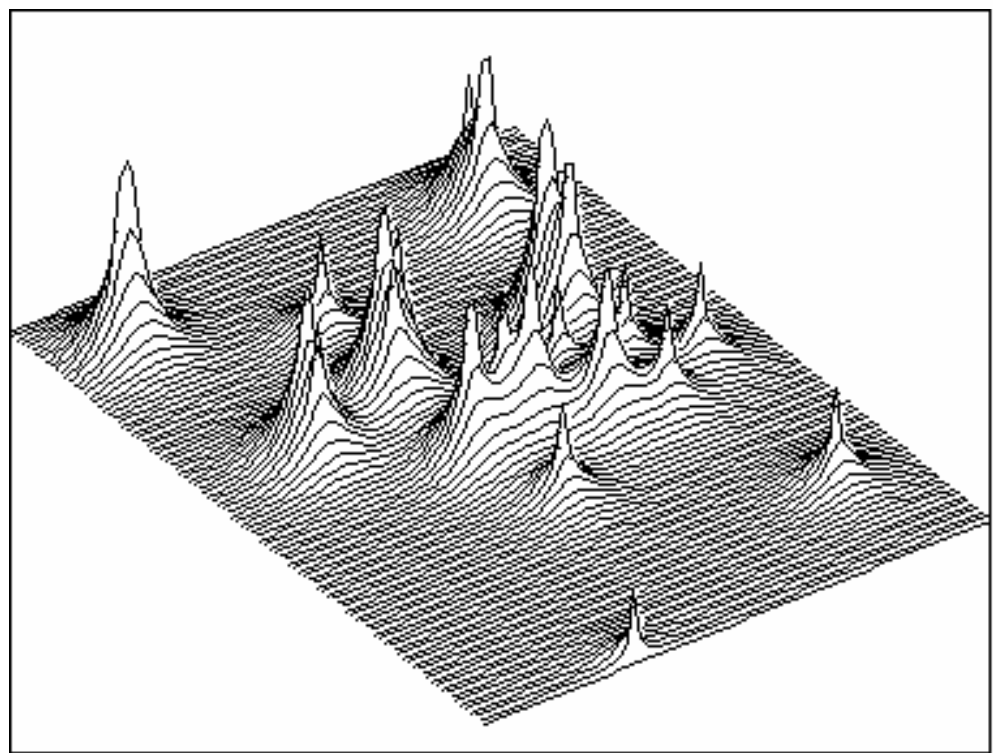

Figure 2. The initial surface $\mathrm{D}(\mathrm{i}, \mathrm{j})$ for site fp 20

We applied a threshold of 0.4 to the data from Figure 1 to get the target array R. Figure 2 shows the diffusion surface computed with the Equation (3), when the input to the diffusion process is target array R, computed from the data of Figure 1.

Initially, the diffusion surface is flat - all values the same, a small positive number (we frequently used 0.001). Then, the diffusion surface is computed with the above equations, for a fixed number of iterations, by considering that the target position matrix is constant in time. In the following, we'll call this process "diffusion surface setting" (DSS).

After DSS we introduce the demining agent that begins to move toward the nearest target position. If no mine is found, the diffusion value, from of the current agent position, is zeroed during each move. After the position of the first target is found, the corresponding element from the matrix $\mathrm{R}$ is zeroed, and another DSS is made. Starting from the actual position, the agent will move to the nearest target position. If the agent finds a mine at the current

position, the corresponding diffusion value is made one.

Every "find" of a target position is accompanied by a DSS process. Thus, the target positions are successively found and eliminated from the agent "target list".

Figure 3 shows the movement path of the animat, corresponding to the diffusion surface of Figure 2. For all the enclosed simulations, the agent start position is at the bottom left corner of the animat movement plane. The targets are represented as small black rectangles, and the agent (animat) path with a continuous line. The diffusion array had the dimensions $75 \times 100$. We used 180 iterations for every DSS. After 334 movements the animat visited all the target positions.

The process of target-position finding has two steps:

1) search for the largest diffusion value, namely the $D(i, j)$ value, in the current neighborhood;

2) movement of the agent to the adjacent cell with highest $\mathrm{D}(\mathrm{i}, \mathrm{j})$ value.

Barriers. Our model allows for barrier avoidance. Barriers exist and are described by a matrix, the barrier array, B, which has the binary elements $b(i, j)=1$, if $(i, j)$ is a barrier point, and $b(i, j)=0$ otherwise. For every $(i, j)$ that is a barrier point, we transform all its neighborhood points into barrier points.

If the agent can't move to a location (because of the barrier), the diffusion connection between the current position and the moved-to position is cut. We implemented this process through a connection matrix $\mathrm{C}$ with the dimension $\mathrm{M} \times \mathrm{N} \times 8$, and having the elements $c(i, j, n)$. The element $c(i, j, n)=1$ if movement between position $(i, j)$ and its neighbor $n$ is possible, and $c(i, j, n)=0$ otherwise.

Taking into account the connection matrix $\mathrm{C}$, the Equation (1) becomes:

$$
D_{k}(i, j)=D_{k-1}(i, j)+\alpha \sum_{V} c_{V, k-1}(i, j)\left[D_{V, k-1}(i, j)-D_{k-1}(i, j)\right]+r_{k-1}(i, j)
$$

where $c_{V, k}(i, j)$ represents the elements from the matrix $\mathrm{C}$, corresponding to the neighborhood of the current agent position $(i, j)$. 


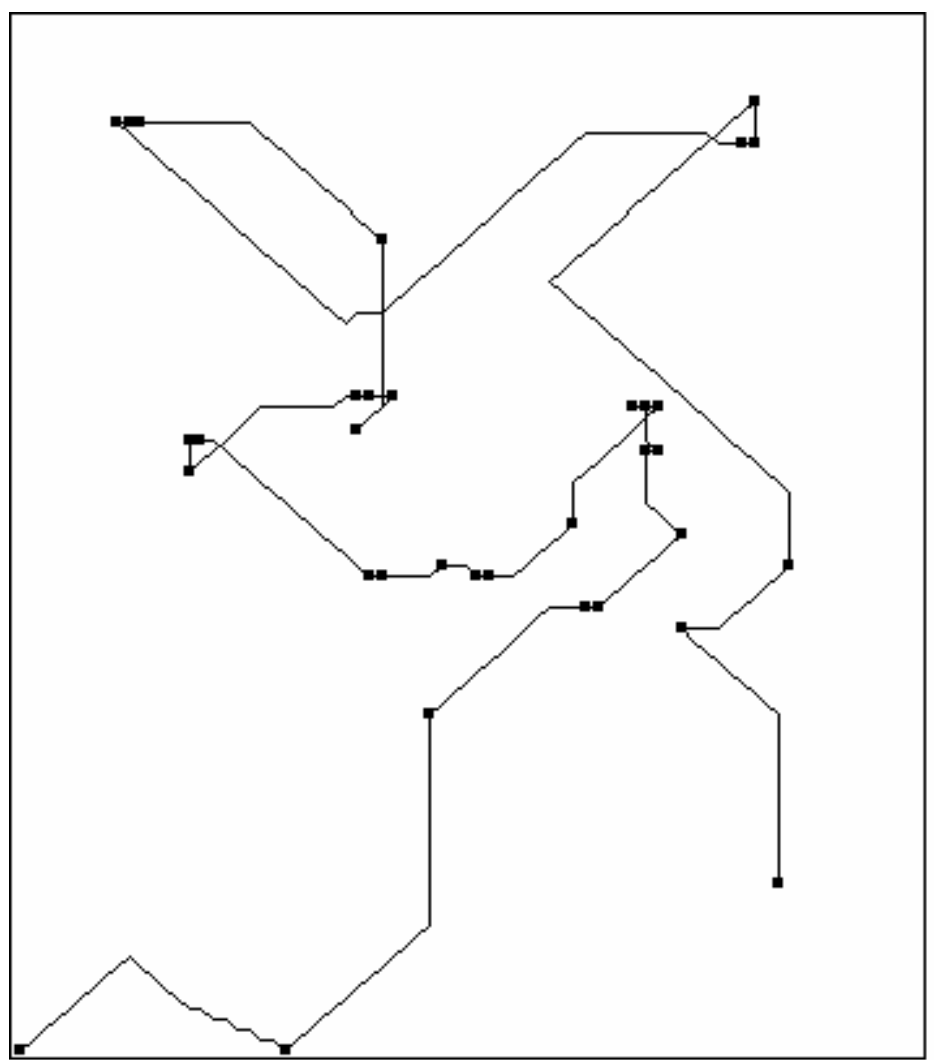

Figure 3. Animat mowement path for site fp20
Initially all elements of the connection matrix $\mathrm{C}$ are equal to one. When the agent chooses the next move, it also tests, by using the matrix barrier $\mathrm{B}$, if the position of the next movement is a barrier point. If it is so, the corresponding connection matrix element is made zero, and another possible movement is tested. This way the matrix $\mathrm{C}$ is updated during the agent movement, such that barrier points are never reached. We can say that the connection matrix $\mathrm{C}$ represents the animat's knowledge about the barrier map B.

Before the agent discovers a barrier point, it is attracted to that point through the diffusion process. After the connection to the barrier point is broken, the diffusion value at the barrier point is zeroed permanently. Thus, that point will never be visited by the agent, because of the way we implemented the barrier map B. The points of the barriers are discovered sequentially, while the agent is making in fact a detour of the barrier.

Figure 4 shows the movements of the agent when a barrier exists in the movement plane, from figure 3 . The agent needs 935 movements to make barrier detours and to visit all the target positions.

If all the agents follow the same diffusion surface (which implies some central controller), but have different starting points or times, there is no possibility that they will interfere with each other. This happens because the animat movement to a cell is accompanied by forcing to zero the mine probability (diffusion value) of the cell. Thus, if an agent moves to a cell, another agent will not attempt to move to the same cell, because the cell mine probability has already been zeroed. Note that if any individual animat is destroyed or ceases to function, guidance of the other animats is unimpaired.

\section{COMPUTER SIMULATIONS}

The computer simulations were made with two programs developed by the authors: one program written in BorlandC for sensor data pre-processing and a program written in VisualC ++5.0 under WinNT 4.0 for the diffusion-based spatial navigation.

The BorlandC program first transforms the original data, recorded as text files, in real numbers. Then, it pre-processes data (transforms the sensor data in LSR scaled data) and save them as an array of real numbers.

Starting with the array of the LSR data, the VisualC++ program makes the rest of the computations and displays different results of the demining application.

We used sensor data from four sites: fp20, fp22, 61sb and 61tc. All the data were recorded with the EM61 (a high sensitivity metal detector). In all simulations, we used only the data taken from the top coil of the detector.

In the computer simulations we initialized the diffusion surface to $D_{0}(i, j)=0.001$, for all $i$ and $j$. We used a diffusion coefficient $\alpha=0.05$ for the simulations included in this presentation.

In Figures 5 and 6 we represent the initial diffusion surface (before the animat begins to move) and the movement plane for the data taken from site fp22. The site dimensions were $30 \mathrm{~m} \times 100 \mathrm{~m}$. The agent used 129 movements to visit all the targets.

The number of movements needed for the detecting all the targets is computed for every experiment. If all targets are actual mine locations (an ideal situation), this number can be interpreted as a performance measure. We leave until later a more accurate computation of performance that would include not just the search time, but would also take into account misses (undetected mines), false alarms (unsuccessful digs) and the relative costs of each of these. 


\section{CONCLUSIONS}

Our initial computer simulations lead us to conclude that diffusion-based route-finding is an efficient and simple method for using multiple autonomous agents to search for objects of positive or negative value, such as food (for animals) or mines (for demining).

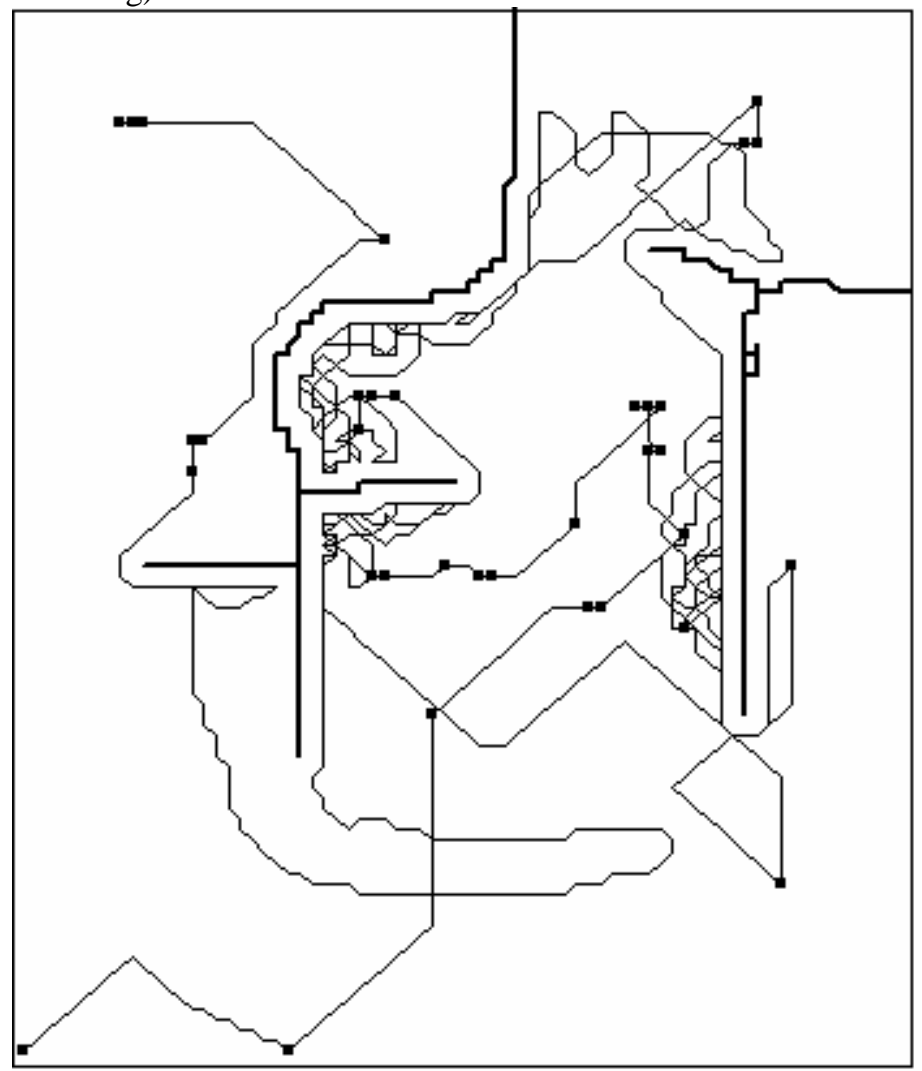

Our initial experiments suggest that:

- Searching for multiple targets, in open terrain, a diffusion-based guidance is efficient, if not completely "shortest-path" *.

- Some of the inefficiency derives from our digital approximation to continuous diffusion. We are currently exploring ways to minimize this source of inefficiency.

- Searching for multiple targets, on a terrain with barriers, is safe (all targets are finally found) but the searching time increases, with respect to the open-field case.

The accuracy of any guidance system obviously depends critically on the accuracy of the processed sensory data. We used for a target matrix obtained from singlesensor LSR data. A more accurate target matrix might be obtained given more sophisticated sensor-data processing 18

We also plan to explore modified versions of diffusion that can deal with different constraints on the target distribution and explore their implications for different value functions.

Figure 4. Light line: Animat path for site fp20. Heavy line: Barriers

\footnotetext{
* Note that "shortest path" is not necessarily optimal for either foraging or mine detection. Because delayed food is discounted relative to present food, the optimal foraging path will not always be the shortest; it will generally be the path that assures the highest immediate rate of food acquisition. For example, an animal (and the diffusion model) might prefer to deplete a high-density resource at a distance before exploring a low-density resource close by - even though the opposite strategy would have a shorter total path length. Conversely, in the case of mines it may be more important to identify a mine-free path, or to miss a minimum number of mines in a given area, either of which would lead to objective functions different from shortest-path. We believe that the diffusion approach can be tuned to accommodate different objective functions.
} 


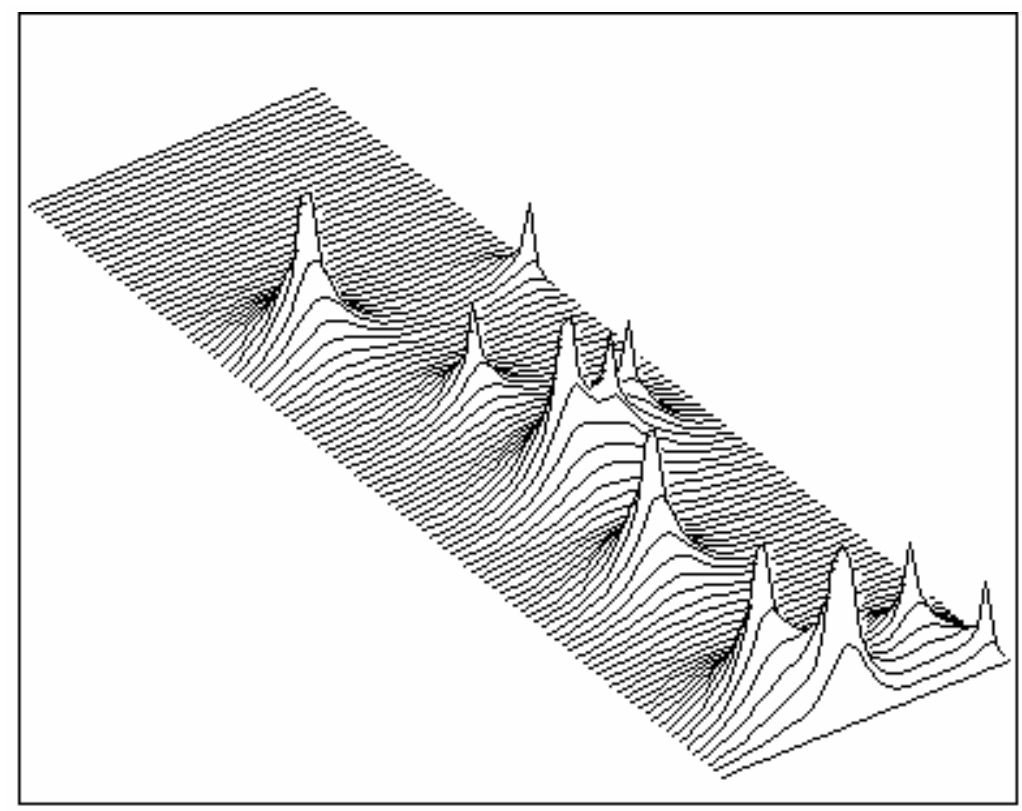

Figure 5. The initial surface D(i,j) for site fp22

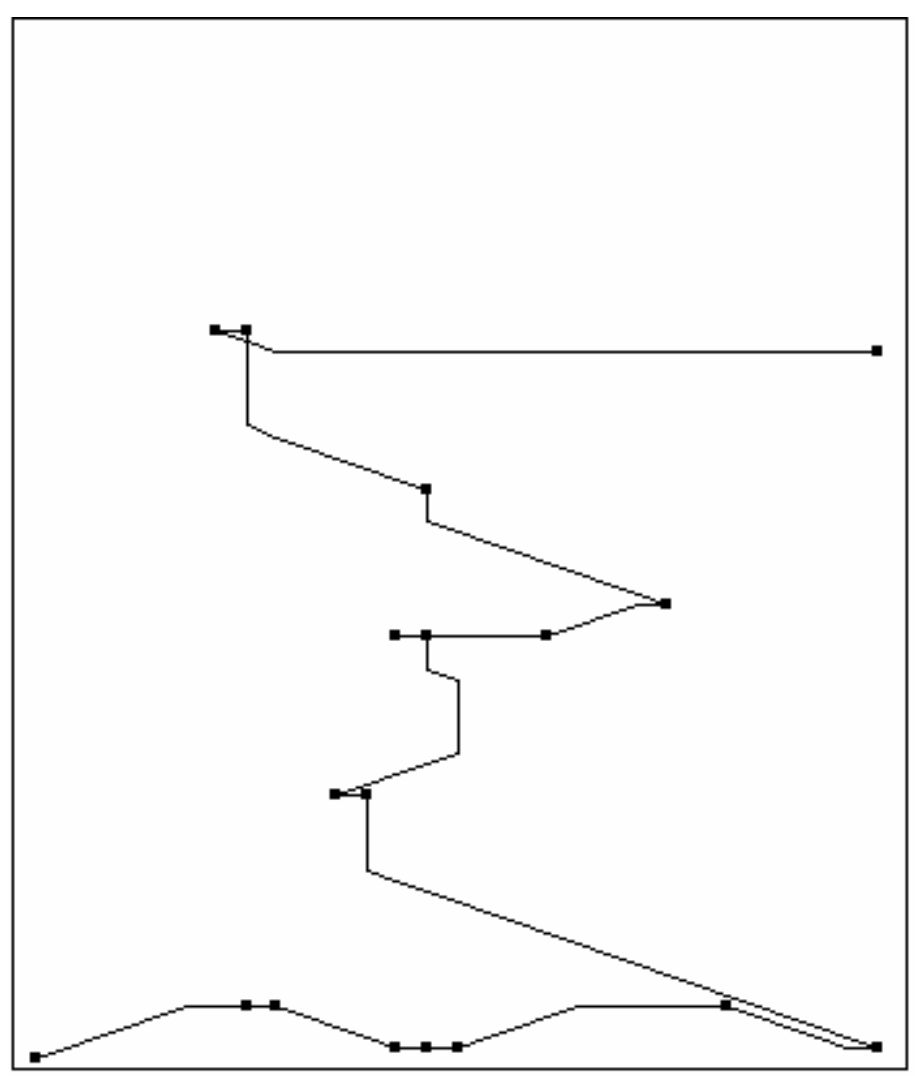

Figure 6. Animat movement path for site fp22 


\section{ACKNOWLEDGEMENTS}

This work was supported by U.S.Army Research Office, Grant No. DAAH04-96-1-0448 (Multidisciplinary University Research Initiative to Demining).

\section{REFERENCES}

1.J. E. R. Staddon and A. K. Reid, On the dynamics of generalization, Psychological Review 97, pp.576-578, 1990

2.A. K. Reid and J.E.R. Staddon, A Dynamic Route-Finder for the Cognitive Map, paper submitted to Psychological Review

3.D. Maio and S. Rizzi, Learning and clustering in autonomous systems, IEEE Transaction On Pattern Analysis and Machine Intelligence, Vol. 15, no.12, pp.1286-1297, 1993

4.E. Hou and D. Zheng, Mobile robot path planning based on hierarchical hexagonal decomposition and artificial potential fields, Journal of Robotic Systems, pp.605-614, 1994

5.C.R.Weisbin, G.de Saussure, J.R.Einstein and F.G.Pin, Autonomous mobile robot navigation and learning, Computer, pp.2935,1989

6. J.D.Carthy, Animal Navigation, Charles Scribner's Sons, New York, 1956

7. O.Trullier, S.I.Wiener, A.Berthoz and J.A.Meyer, Biologically based artificial navigation systems: review and prospects, Progress in Neurobiology, Vol.51, pp.483-544, 1997

8. M.Muller and R.Wehner, The hidden spiral: a systematic search and path integration in desert ants, Cataglyphis fortis, J.Comp. Phsiology A175, pp.525-530, 1994

9.G.Hartman and R.Wehner, The ant's path integration system: neural architecture, Biological Cybernetics 73, pp.483-497, 1995

10.T.S.Collet and J.Baron, Biological compasses and the coordinate frame of landmark memories in bees, Nature 368, pp.137-140, 1994

11.S.Benhamou, P.Bovet and B.Poucet, A place navigation algorithm based on elementary computing procedures and associative memories, Proc. of the Third Int. Conf. on Adaptive Behavior, MIT Press Bradford Books MA, pp.206- 213, 1994

12.T.S.Collet, The use of visual landmarks by gerbils: reaching a goal when landmarks are displayed, J.Comp.Pysiol. A 160, pp.109-113, 1987

13.D.M.Wilkie and R.Palfrey, A computer simulation model of rat's place navigation in the Morris water maze, Beh. Res Math. Instrum.Comput. 19, pp.400-403, 1987

14.N.Schmajuk, A.D.Thieme and H.T.Blair, Maps, routes and the Hippocampus: A neural network approach, Hippocampus, Vol.3, No.3, pp.387-400, 1993

15.N.Schmajuk and H.T.Blair, Place learning and the dynamics of spatial navigation: A neural network approach, Adaptive Behavior, Vol.1, No.3, pp.353-385, 1993

16.EM61 High Sensitivity Metal Detector, Operating Manual, Geonics Limited, Mississauga, Ontario, March 1995

17.Press et al, Numerical Recipes in Pascal, Cambridge Univ. Press, 1989.

18. E. Gelenbe and T. Kocak, Area-based Results for Mine Detection, Research Report, Duke Univ., 1997. 